\title{
Assessing the effectiveness of enhanced psychological care for patients with depressive symptoms attending cardiac rehabilitation compared with treatment as usual (CADENCE): study protocol for a pilot cluster randomised controlled trial
}

Suzanne H. Richards ${ }^{1 *}$, Chris Dickens ${ }^{1}$, Rob Anderson ${ }^{1}$, David A. Richards ${ }^{1}$, Rod S. Taylor ${ }^{1}$, Obioha C. Ukoumunne², David Kessler ${ }^{3}$, Katrina Turner ${ }^{4}$, Willem Kuyken ${ }^{5}$, Manish Gandhi', Luke Knight ${ }^{6}$, Andrew Gibson ${ }^{7}$, Antoinette Davey ${ }^{1}$, Fiona Warren ${ }^{1}$, Rachel Winder ${ }^{1}$, Christine Wright ${ }^{1}$ and John Campbell ${ }^{1}$

\begin{abstract}
Background: Around $17 \%$ of people eligible for UK cardiac rehabilitation programmes following an acute coronary syndrome report moderate or severe depressive symptoms. While maximising psychological health is a core goal of cardiac rehabilitation, psychological care can be fragmented and patchy. This study tests the feasibility and acceptability of embedding enhanced psychological care, composed of two management strategies of proven effectiveness in other settings (nurse-led mental health care coordination and behavioural activation), within the cardiac rehabilitation care pathway.
\end{abstract}

Methods/Design: This study tests the uncertainties associated with a large-scale evaluation by conducting an external pilot trial with a nested qualitative study. We aim to recruit and randomise eight comprehensive cardiac rehabilitation teams (clusters) to intervention (embedding enhanced psychological care into routine cardiac rehabilitation programmes) or control (routine cardiac rehabilitation programmes alone) arms. Up to 64 patients (eight per team) identified with depressive symptoms upon initial assessment by the cardiac rehabilitation team will be recruited, and study measures will be administered at baseline (before starting rehabilitation) and at 5 months and 8 months post baseline. Outcomes include depressive symptoms, cardiac mortality and morbidity, anxiety, health-related quality of life and service resource use. Trial data on cardiac team and patient recruitment, and the retention and flow of patients through treatment will be used to assess intervention feasibility and acceptability. Qualitative interviews will be undertaken to explore trial participants' and cardiac rehabilitation nurses' views and experiences of the trial methods and intervention, and to identify reasons why patients declined to take part in the trial. Outcome data will inform a sample size calculation for a definitive trial.

Discussion: The pilot trial and qualitative study will inform the design of a fully powered cluster randomised controlled trial to evaluate the effectiveness and cost-effectiveness of the provision of enhanced psychological care within cardiac rehabilitation programmes.

(Continued on next page)

\footnotetext{
* Correspondence: s.h.richards@exeter.ac.uk

'University of Exeter Medical School, St Luke's Campus, Exeter, EX1 2LU, UK

Full list of author information is available at the end of the article
} 
(Continued from previous page)

Trial registration: ISRCTN34701576 (Registered 29 May 2014)

Keywords: Depression, Coronary heart disease, Multimorbidity, Behavioural activation, Mental health care coordination, Cardiac rehabilitation, Randomised controlled trial, Qualitative interviews

\section{Background}

Prevalence of depression and associated outcomes for patients with acute coronary syndromes

Major depression is common among people with coronary heart disease. Most research has focused on patients with acute coronary syndromes (ACS), among whom prevalence rates for depression are around $20 \%$ as determined by studies using rigorous clinically structured research interviews [1]. Rates of depression have also been shown to be elevated in individuals following coronary artery bypass grafting [2], patients with unstable angina [3] and patients with chronic heart failure [4]. These rates greatly exceed those seen in the UK general population (2.6\% [5]), suggesting that the associations between coronary disease and depression may be causal (direct or indirect).

The nature of the association between depression and coronary disease is complex. Depression can predate an ACS, approximately doubling the risk of subsequent incident ACS [6, 7], and worsen associated heart failure [8] or cardiac mortality [9]. Depression that predates an ACS is predicted by younger age, female sex, lack of social support, ongoing life difficulties and a past psychiatric history [10]; these are similar to risk factors for depression in the general population. Depressive symptoms may occur after an ACS, so-called 'new onset' depression [10]; approximately $20 \%$ of individuals after a recent myocardial infarct $(\mathrm{MI})$ develop depressive symptoms in the 12 months following their cardiac event. 'New onset' depression is not associated with the usual risks for depression, but is predicted by negative illness representations relating to the ACS and ongoing cardiac symptoms $[10,11]$. Mechanisms underpinning the association between depressive symptoms and poor cardiovascular outcomes may include biological and behavioural processes, or may be confounded by shared genetic vulnerability, environmental stresses, or perseverative negative cognitive processes [12].

Depression among people with an established ACS is an important predictor of negative medical outcomes including poorer health-related quality of life [13, 14], greater morbidity and mortality [15-18], greater use of routine and unscheduled health care $[19,20]$ and greater health care costs [21]. Some studies have indicated that the timing of onset of depressive symptoms with regards to cardiac-related hospitalisation may be important in determining health outcomes. In particular, 'new onset' depression that develops following an MI has the greatest association with subsequent mortality [21-24]. It remains unclear, however, whether 'new onset' depression is particularly 'cardiotoxic' or whether its apparent associations with poor cardiac outcomes is confounded [23] by, for example, the severity of the underlying cardiac disease or due to a retention bias, with patients suffering with premorbid depression dying before reaching follow-up [9].

\section{Usual care for patients with cardiac disease and depression}

In the UK, the NHS routinely offers multidisciplinary cardiac rehabilitation $(\mathrm{CR})$ to patients who experience an acute cardiac event. CR follows a seven-stage standard patient care pathway (stages 0-6, Fig. 1) [25, 26]. Guidance on the recommended content of standard care is published by the British Association for Cardiovascular Prevention and Rehabilitation (BACPR) [26]. Core components include: education; exercise and physical activity; diet and weight management; medical management; and psychological support. In 2010-2011 a national CR audit [27] identified 290 operationally distinct, locality-based comprehensive cardiac rehabilitation programmes (CCRPs; stages 2-5). These teams undertake patient assessments (including mental health status), then generate and implement CR care plans.

Patient attrition across the CR pathway is high. Although around $17 \%$ of patients screen positive for depressive symptoms when attending an initial, locality-based CCRP assessment (stages 2-3), around half of these will not proceed to CCRP. Despite the burden of depressive symptoms, only $10 \%$ of CR services report direct psychology input [27] and patients' depression usually remains untreated [28]. Locally agreed referral protocols providing for access to psychological care at either the tertiary (stage 1) or community (stages 2-5) level may be agreed. However, the precise content of such protocols and the consistency of their implementation remain unclear from audit data.

While it would appear that structured management of depressive symptoms is not routinely provided in CR, patients can access mental health care through existing care pathways for depression [29-31]. Effective care includes mental health care coordination, which is often 


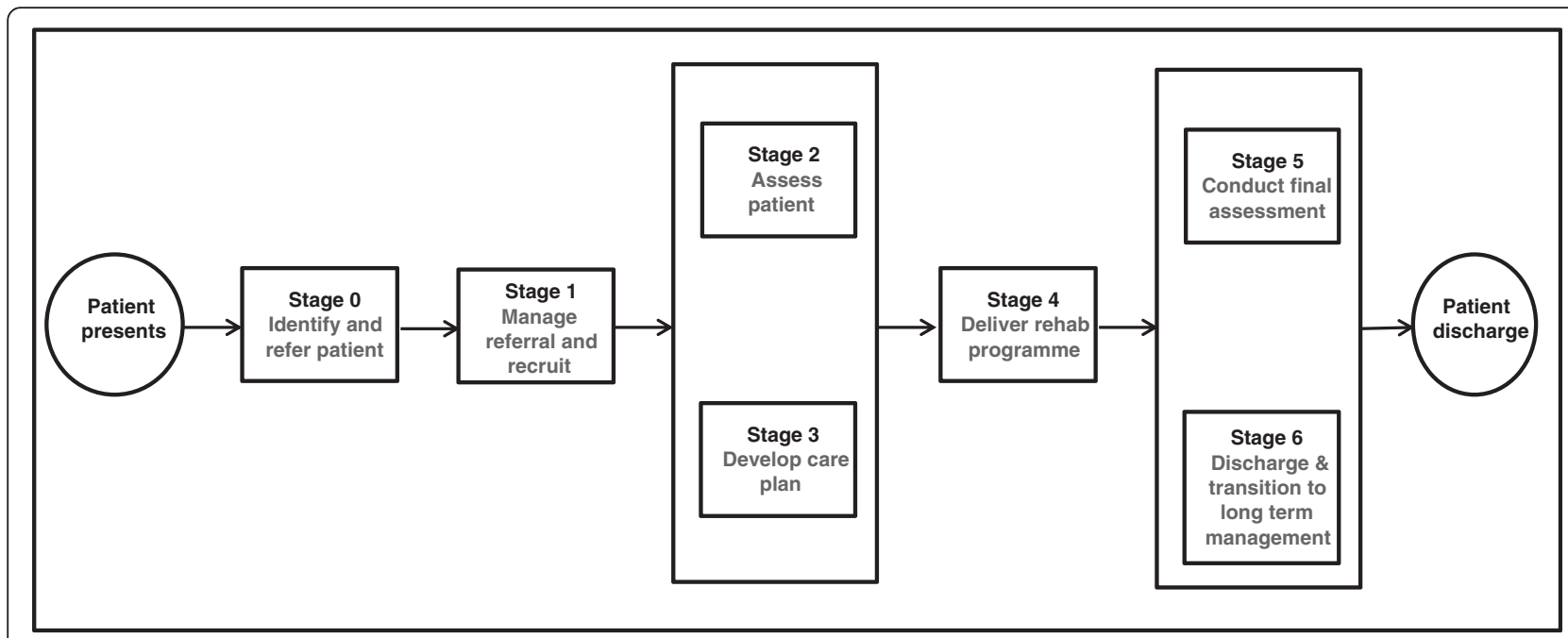

Fig. 1 UK cardiac rehabilitation care pathway. UK Department of Health's '0-6 Stage Patient Pathway of Care' for cardiac rehabilitation services [83]

managed in primary care and involves the use of stepped care algorithms, and access to psychological or pharmacological interventions of proven effectiveness.

In general populations, cognitive behavioural therapy $(\mathrm{CBT})$ and antidepressant medication (ADM) are the two treatments with most evidence of effectiveness, and both are recommended by the National Institute for Clinical Excellence (NICE) [29, 30]. However, there are problems with ADM, which include side-effects, poor patient adherence and relapse risk on ADM discontinuation. Service-user organisations and policy think tanks advocate greater availability of psychological therapies, which many people prefer [32]. CBT, which is of similar efficacy to ADM [33], has several advantages: it is consistent with many service users' preferences for nonpharmacological treatment, and it modifies the illness trajectory as its benefits continue after the end of treatment by teaching skills to prevent depressive relapse in the long term. However, CBT has several potential disadvantages. Not all patients may engage or adhere to CBT, due to a multiplicity of reasons (e.g. burden of homework, feelings of confusion or upset at revisiting painful or difficult emotions and situations during therapy) [34]. CBT requires extensive training to deliver competently, and is typically offered only by specialist CBT therapists. The high costs of training and employing sufficient therapists may limit attempts to access CBT. The recent 'Improving Access to Psychological Therapies' (IAPT) $[35,36]$ programme in England has done much to enhance access and recovery rates which are at approximately $50 \%$. However, IAPT does not reach into specialist CR settings where brief and accessible psychological treatments may have much to offer.

One such psychological treatment is that of Behavioural Activation (BA). BA is postulated to alleviate depression by focusing directly on changing behaviour based on behavioural theory [37]. This theory states that depression is maintained by avoidance of normal activities. As people withdraw and disrupt their basic routines, they become isolated from positive reinforcement opportunities in their environment. They may then end up stuck in a cycle of depressed mood, decreased activity and avoidance [37]. BA systematically disrupts this cycle, initiating action in the presence of negative mood, when people's natural tendency is to withdraw or avoid [38]. Although CBT incorporates some behavioural elements, these focus on initiating behavioural experiments to test specific beliefs. In contrast, BA targets avoidance from a contextual and functional approach not found in CBT, focusing on understanding the function of behaviour and replacing it accordingly. BA also explicitly prioritises the treatment of negatively reinforced avoidance and rumination. Furthermore, the BA rationale is easier to understand and operationalise for both patients and mental health workers than CBT, where activity is also increased but the primary techniques focus on changing maladaptive beliefs [39]. Its focus on context and functioning is also closely aligned to the ethos of $C R$ services. The relative simplicity of BA treatment makes it relatively straightforward to train nurses without mental health training in its application [40-42]. The study team includes the expertise in respect of the design, training, and delivery of a BA-based intervention [41-43].

\section{Rationale for current research}

There is a lack of clear evidence of efficacy for conventional treatments for depression in patients with ACS. Trials of the antidepressants sertraline, citalopram and mirtazapine have shown small or no effect on depression among patients with ACS [44-46]. Efficacy of 
psychological treatments, which are usually preferred by patients, remains unclear. Recent systematic reviews $[47,48]$ report modest but significant reductions in depression for patients with coronary disease receiving psychological treatment, although the methodological quality of studies selected is such that it precludes firm conclusions regarding which treatments are most effective. Several high-quality randomised controlled trials (RCTs) in patients with ACS and depression have not confirmed the benefits of psychological therapy in this population. In the large ENRICHD trial, CBT plus citalopram led to very small improvements in depression that were not sustained [49]. Similarly, the CREATE trial found that interpersonal psychotherapy was no better than standard care [44]. In contrast, the CODIACS Vanguard trial RCT $[50,51]$ found that a patient preference intervention (management options available included problem-solving therapy delivered by a centralised service offered via phone or the web, ADM, both or neither) combined with a stepped care algorithm significantly improved depressive symptoms at 6-months post randomisation. However, these results must be interpreted with caution as the sample size was small $(n=150)$, and no conclusions can be drawn regarding the effectiveness of individual therapeutic options available as part of the preference intervention. No trials to date have evaluated the effectiveness of BA in patients with an ACS and 'new onset' depression.

Most intervention studies have recruited participants immediately following an acute cardiac event, and the complexity of depression in that period may have contributed to the lack of treatment response. The reasons for the limited benefits of conventional depression treatments in patients with coronary disease are not clear. First, depression that starts after an ACS is different from depression in the general population. PostACS depression does not have the usual risk factors as seen in the general population, but is associated with ongoing cardiac symptoms, and increased concerns about health $[10,11]$, which may act to reduce the response to conventional treatment [45]. Second, conventional psychological treatments, such as CBT, that encourage recall of past experience and that challenge maladaptive thoughts, may be too traumatic for people who have suffered a recent, serious threat to life. Third, conventional psychological therapies available through IAPT are considered expensive and may not be widely available to patients with CHD. Indeed, a recent qualitative study of patients [52] concluded that management of depression should be embedded within CR teams rather than as another source of referral. UK CR programmes experience significant patient attrition at each stage of the rehabilitation pathway, with over half of patients dropping out before locality-based CCRP commences. Given this attrition, for the $17 \%$ of patients with depressive symptoms who actually attend at least one assessment with a CCRP, 'another outward referral' for psychological care may simply be a further barrier to accessing timely care. If a low-intensity psychological treatment, such as BA, has the potential to be safely and effectively [38, 40-42, 53, 54] embedded within the CCRP, this could holistically tackle the depressive symptoms at the same time as physical rehabilitation as part of patient case management. Similarly, acknowledging that attrition from rehabilitation is high even in patients who attend an initial CCRP assessment, we also believe that any attempt to enhance psychological care should include training CR nurses (who routinely screen for depressive symptoms) to apply evidence-based referrals for those who elect not to attend rehabilitation. Such mental health care coordination will also be in place for patients completing CCRP (including $\mathrm{BA})$, but whose symptoms do not respond.

\section{Study aims and objectives}

The overarching aim of this pilot study is to test the methods and procedures required to undertake a fully powered evaluation of the clinical effectiveness and costeffectiveness of $\mathrm{CR}$ teams implementing an enhanced psychological care intervention (EPC) for patients with 'new onset' depressive symptoms using CR services compared with CR services delivering usual care. Specific objectives are:

1. To quantify the flow of patients (i.e. eligibility, recruitment and attrition rates) from the cardiac event to the 8-month follow-up for patients entering the CR care pathway, and in particular, to document the flow of those patients who agree to take part in the pilot trial

2. To collect participant outcome data in order to estimate the standard deviation for continuous outcomes to inform sample size calculations for a definitive trial

3. To establish the data collection methods required to support a definitive economic evaluation

4. To gather qualitative evidence from patients (including participants who did or did not adhere to the EPC, and those who declined to take part in the trial) and CR nurses on the acceptability of EPC, and on the appropriateness of study methods and procedures. Information will also be sought on the content of usual psychological care within CR teams

\section{Methods/Design}

Consistent with Medical Research Council (MRC) guidance [55] a two-phase study was planned, including a mixed-method feasibility study, followed by a pilot 
cluster randomised controlled trial (RCT) and nested qualitative interview study.

\section{Pre-pilot feasibility study}

Although the focus of this paper is to report the design of the pilot trial, a brief summary of the feasibility study is provided here. The feasibility study (completed March 2015 prior to commencing the pilot trial) employed a mixed-method design to develop and assess an EPC intervention and to undertake preliminary testing of its implementation. Observations of usual care, a before and after observational study, and qualitative interviews with staff and service users were undertaken. The EPC intervention was developed working with four nurses from three NHS CCRP services (stages 2-5; see Fig. 1) from South West England. Nine eligible patients (i.e. individuals with 'new onset' depression) were recruited, and both staff and patients were invited to comment on the feasibility and acceptability of implementing and/or experiencing EPC. Additional CR process data was recorded, including participant attendance at, and adherence with the CCRP with embedded EPC. The findings from this feasibility study were used to refine and develop the EPC intervention and the pilot trial methods. Specifically, qualitative and observational data, considered along with findings arising from discussions with lay representatives, identified the need to reduce the intensity of the BA component of the EPC delivered by CR nurses operating within the context of routine care. The EPC intervention to be delivered in the pilot study will thus retain the nurse acting as a mental health care coordinator, using clearly defined clinical decisionmaking points and applying evidence-based referral pathways, with the BA component delivered as a participant-led BA intervention, actively supported by the CR nurse.

\section{Pilot cluster randomised controlled trial}

An external pilot cluster RCT $[56,57]$ will be undertaken, including the piloting of economic data collection, and a qualitative interview study. A Consolidated Standards of Reporting Trials (CONSORT) diagram describing the flow of participants through the study is presented in Fig. 2.

\section{Trial interventions}

\section{Usual care (control) intervention}

Usual care is defined here as the standard CR rehabilitation commencing at the point of assessment undertaken by a CR specialist nurse from a locality-based CCRP (stages 2-3). Of patients invited to attend CCRP, around $41 \%$ attend structured sessions within 2-10 weeks (stage 4) [27]. Treatment as usual in CCRPs typically includes intensive rehabilitation for 1-2 sessions per week for approximately 8 weeks. Sessions generally last around
2 hours and include structured exercise, education (e.g. managing lifestyle and cardiac risk) and some psychological input (e.g. relaxation, stress management) in order to meet the core standards of care described by BACPR [26]. On exiting CCRP, a final assessment (stage 5) and discharge arrangements will be made to community services (stage 6).

There is debate as to what constitutes standard psychological care within CR [27], and a core objective is to clarify this definition. National audit data found that psychological expertise within locality-based CCRPs is uncommon $(<10 \%)$. In the feasibility study, some patients with depressive symptoms were referred to a clinical psychologist. However, no systematic approach was routinely adopted to identify patients as being suitable for referral. The decision was pragmatic, being based on a combination of reviewing depression scores obtained in standard CCRP assessments, discussions with patients, and clinical judgment regarding who might benefit from referral. Informal discussions with mental health specialists and CR staff suggest that there is considerable variation in local CCRP protocols across the UK.

\section{Enhanced psychological care (treatment) intervention}

Based on our feasibility findings, we propose to embed EPC within existing CR care pathways, with referrals to existing community mental health services as appropriate. CR nurses from operationally distinct 'clusters' locality-based CCRPs (see Fig. 1, stages 2-5) will be trained to deliver EPC based on a nurse training manual developed and refined during the feasibility study. The CR nurse will implement mental health care coordination $[29,30]$, including an embedded participantled BA programme [38, 40-42, 53, 54]. Pre-existing participant and nurse training materials used by the coapplicant (DAR), in previous research, were adapted during the feasibility phase following an iterative process of qualitative data collection with trainers, CR nurses and patients, combined with lay and clinical advisors' feedback.

To deliver mental health care coordination, CR nurses will apply clinical decision-making rules based on current best practice $[29,30]$, matching the intensity of treatment with participant preferences for mental health care. All patients referred to a locality-based CCRP and who agree to undertake an initial assessment (stages 2-3), will be routinely screened for depressive symptoms using the Patient Health Questionnaire (PHQ-9) [58, 59]. This tool assesses the nine Diagnostic and Statistical Manual of Mental Disorders (DSM-IV) depression criteria, with individuals scoring 10 or more (out of a possible score of 27) during their initial CR assessment deemed eligible for inclusion. 


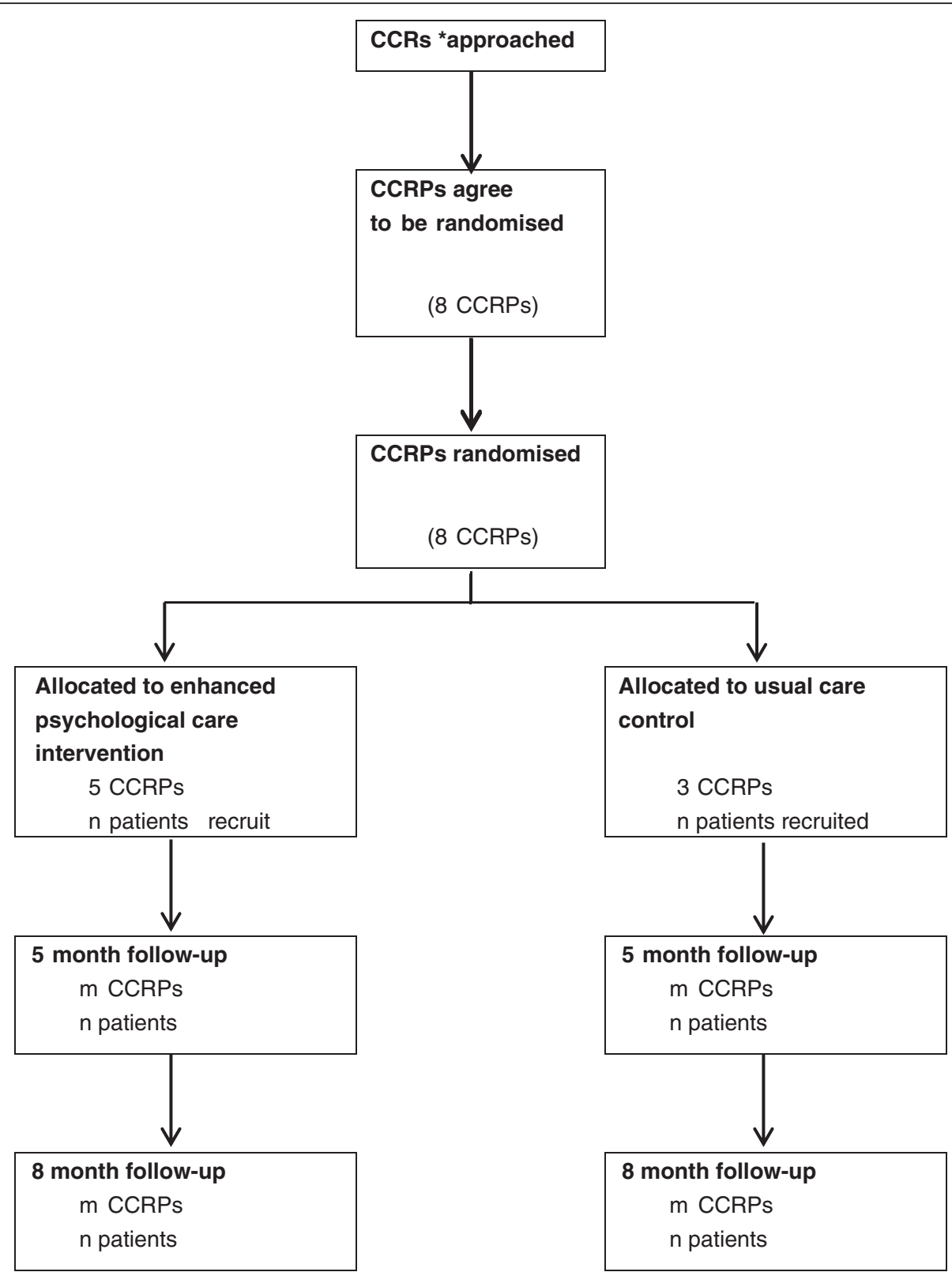

Fig. 2 Consolidated Standards of Reporting Trials (CONSORT) diagram. CONSORT diagram (with cluster extension) for the CADENCE pilot randomised controlled trial (RCT)

On identifying an eligible individual, the nurse will explain what evidence-based treatment options are available. This includes BA self-help materials supported by the nurse, GP referral, referral to local IAPT services, and/or referral to specific cardiac patient psychological support services where available. Nurses will coordinate care by monitoring symptoms of depression and anxiety, assessing risk to self or others and agreeing a plan of care with participants that may include BA and/or referral to GP, IAPT or other therapeutic agencies as described above.
As part of this care coordination protocol, all participants will be offered nurse-supported self-help using a participant BA manual. The self-help BA manual consists of a structured programme aimed at enabling participants to re-engage with sources of positive reinforcement from their environment and to develop future strategies for managing their depressive symptoms. The manual adopts a functional analytical approach, aiming to help the participant develop an understanding of behaviours that might interfere with meaningful, goal-oriented behaviours (e.g. negative avoidant behaviours). The manual also explains 
how to self-monitor mood, and then to identify patterns of behaviour associated with their depression. Participants are encouraged to develop alternative behaviours which are goal- orientated, targeting routine, pleasurable, and necessary activities, and activity scheduling of these identified behaviours.

Although our pilot intervention involves a participantled self-help BA manual, the CR nurses will actively support participants. The $C R$ nurses will attend a 2-day training course delivered by experienced mental health practitioners, during which they will receive training on the key techniques of mental health care coordination, managing safety and risks, and BA. This training will allow nurses to actively support participants during care coordination and as they work through the BA manual alongside their CR programme. Nurses will also be provided with a session-by-session guide, detailing the types of care coordination tasks which might be relevant for participants as they move through their 6-8 week structured programme (Additional file 1). This guide is flexible to accommodate participant preferences for care. Nurses who are delivering EPC to study participants will also receive bi-weekly clinical supervision by an experienced mental health practitioner.

For those participants who complete CCRP (stage 5), structured details of the care received will be sent to their GP. All participants, including those receiving BA and whose depressive symptoms do not respond, will be given an opportunity to review their management options with the nurse. Here, treatment response is defined as achieving a minimally important clinical difference (MICD) for the PHQ-9 equivalent to a 5-point reduction in score [60]. It is important to note, however, that some people who achieve such a MICD may remain above the PHQ-9 diagnostic threshold $(\geq 10)$. As part of their care coordination, these individuals will be referred for continuing mental health management.

\section{Randomisation and allocation concealment}

An external pilot cluster RCT $[56,57]$ will be undertaken, seeking to recruit and randomise eight CCRPs (clusters) to a treatment or a control arm in a 5:3 ratio. The randomisation ratio was informed by data emerging from our feasibility study, including the need to ensure that sufficient nurses and patients have EPC exposure to support the aims of the qualitative process evaluation. Randomisation will be conducted using the stratifying variables of CR team setting (hospital-based, community-based, or mixed hospital and community), and monthly throughput of new patients assessed for CR at stages $2-3$, facilitating division into small and large teams. Team setting has been selected as a stratifying variable because our feasibility study suggested that teams working in hospital and community settings may encounter different resource issues that could affect the delivery of EPC. Throughput of new patients into $\mathrm{CR}$ services has been selected as a stratifying variable, as this is an indicator of team workload, and will ensure that sufficient participants are recruited into each trial arm.

Randomisation will be carried out by a statistician, independent from the researchers recruiting CR teams, using computer-generated random numbers. A cluster randomised design, as opposed to individual participant randomisation, is essential to avoid contamination between trial arms (i.e. intervention participants coming into direct contact with controls and sharing aspects of their treatment). Our EPC intervention involves training $\mathrm{CR}$ nurses to apply mental health care coordination with embedded participant-led BA. Once trained, it would be very difficult operationally for the nurse to apply structured care coordination to only a subset of eligible participants (as would be required with individual randomisation).

\section{Settings and study population CCRP recruitment procedures}

Eight locality-based CCRPs in South West England will be recruited and randomised. Teams who took part in the feasibility study will be excluded. A two-stage approach to local CCRP recruitment will be employed. We will write to the lead CR nurse in each of the 20 operationally distinct CCRPs in South West England providing detailed information about the study and inviting their participation. In each of the geographical areas the recruitment letter will be co-signed by the trial chief investigator and the local co-applicant. Each team indicating an interest in participation will receive a briefing visit, where the trial design and methods will be explained by the researcher, and the staff will be given an opportunity to ask questions about what might be involved, before being asked to provide written consent to participate in the study.

Nurses in teams allocated to the CCRP with EPC intervention arm will receive EPC and self-harm risk management training (total of two full days) before they start to recruit patients. EPC-trained nurses will be supervised regularly by experienced specialist clinicians/ BA practitioners.

\section{Participant eligibility criteria}

A pragmatic approach will be adopted, whereby adult patients (aged 18 years or over) referred for CR based on local clinical referral protocols will be screened (stages 2-3) for eligibility. The majority will be individuals previously admitted with an ACS (unstable angina, non-ST-elevation MI or ST-elevation MI) or following a coronary revascularization procedure (percutaneous coronary intervention (PCI), coronary artery bypass 
grafting (CABG)), with or without heart failure [61]. Patients with a new episode of depressive symptoms identified through nurse screening at the start of CCRP using the PHQ-9 $[58,62]$ (score 10 or more) are potentially eligible for inclusion. Patient exclusion criteria include: individuals reporting having been actively treated for depression (psychological or drug therapy) within the previous 6 months, or where there is evidence of alcohol or drug dependency, being acutely suicidal, or having poorly controlled bipolar disorder or psychosis/ psychotic symptoms based on a clinical review (seeking external confirmation from the GP or other clinicians as required). No language exclusion will be applied; we anticipate that the majority of patients will have sufficiently good English language skills to engage with EPC. However, consistent with routine practice in delivering psychological therapy, NHS translation resources will be employed to assist participants where required.

\section{Patient participant recruitment procedure}

The CR nurse will apply a structured checklist to ascertain participant eligibility based on our inclusion/exclusion criteria. Eligible participants will be invited to take part in the trial by the CR nurse, and given brief study information to take away and review. The nurse will also ask permission to pass the individual's contact details on to the study researcher. The researcher will then contact the patient within 7 days to discuss study participation and provide them with a detailed Participant Information Sheet. The researcher will arrange to visit the potential participant to obtain written consent and baseline assessment before the participant commences CCRP (stage 4). Potential participants will be reminded of their right to refuse participation, or to withdraw from the study at any time without it affecting their clinical care.

\section{Sample size}

Using UK national audit data (2010-2011), 55,452 patients (for MI, PCI, CABG) undertook phase 3 CR from 280 teams: an average of 200 patients per team per year. Assuming $17 \%$ have depressive symptoms [27], this equates to 35 eligible patients per team each year. The participant consent rate will be established through piloting as no relevant UK data, applicable to cluster trials, are available. Two RCTs (individual randomisation) from the US, exploring psychological/drug therapy in cardiac rehabilitation settings, observed highly variable consent rates of $85 \%(150 / 177$ - Vanguard CODIACS study) [51] and $37 \%$ (2481/6854 - ENRICHD study) [49]. Based on the patient throughput and rates of depressive symptoms observed in our feasibility study, and an assumed participation rate of $50 \%$, we aim to recruit 64 patients through the eight teams in 6 months. This sample size is sufficient to estimate a follow-up percentage as low as $50 \%$ with margin of error $\pm 12.8 \%$ based on the width of the $95 \%$ confidence interval (CI), and as high as $90 \%$ with a margin of error of \pm 9.3 $\%$ based on the lower bound of the $95 \% \mathrm{CI}$. We do not plan to use estimates from the pilot of the intra-cluster (intra-CR team) correlation coefficients (ICCs) for key outcomes to plan the sample size for the definitive trial as the pilot sample is small and estimates will be imprecise. Using national audit data (2010-2011; 6272 patients; 119 CR teams), we estimate the ICC for depression to be 0.047 (95 \% CI: 0.034 to 0.062). This ICC estimate and CI will be used to inform the sample size calculations for the definitive trial.

\section{Data collection}

Participants in both arms will complete baseline measures prior to commencing CCRP, and then two face-toface follow-up interviews with a researcher at 5 and 8 months post randomisation (Fig. 2). Blinding of patients, practitioners or researchers extracting data on study outcomes is not possible in this cluster design, but the final analysis will be carried out by a statistician who is blind to treatment allocation. Data will be collected on process measures, patient-reported outcomes, and resource use and costs.

\section{Process measures}

Process data relating to participant throughput and intervention fidelity will be collected.

To ascertain study eligibility and recruitment we will ask CR nurses to report the numbers of patients: attending an initial CCRP nurse assessment, the proportion identified with depressive symptoms during this assessment, the prevalence of 'new onset' (as opposed to existing) depression and the number of patients who were eligible for study participation. Of eligible patients, we will describe: the number offered study entry; the number who later agreed to be contacted by a researcher; and who subsequently consented to take part and underwent a baseline interview with a researcher. We will also capture data on participant socio-demographic characteristics (age, sex, ethnicity/preferred language) and clinical condition resulting in a CR referral to document how well we have engaged with service users from diverse backgrounds, such as non-English speakers.

Intervention fidelity will be established through a structured notes review documenting the numbers of participants recruited who do not attend CCRP and the proportion with documentary evidence of care coordination in their notes. For participants engaging with the EPC intervention, we will assess adherence by recording the number of BA sessions offered and the number of sessions attended, the number of participants with documentary evidence of care coordination on exiting 
CCRP, and the type of psychological referrals made (if appropriate).

\section{Participant-reported outcomes measures}

Measures are selected on the basis of evidence of their validity in this population, and to ensure coverage of key areas from a clinical and patient perspective (with input from our lay and clinical advisors).

Baseline assessment only This assessment includes some measures that will not be repeated at follow-up. The revised clinical interview schedule (CIS-R) [63, 64] will be administered to ascertain a clinical diagnosis of depression. This score will not determine study eligibility, but will allow its comparison to the other patientreported measures of depression symptom severity ascertained using the Beck Depression Inventory (BDIII) (described below). Some short questions to ascertain, and account for, participant treatment preferences [65] will be included. We will extract data from clinical records (GP, CCRP) relating to known cardiac risk factors (body mass index (BMI), blood pressure, glycosylated haemoglobin (HbA1c), lipids, smoking status) as recorded at the participant's latest clinical assessment prior to recruitment.

Baseline and follow-up measures Although we will request that NHS providers inform us of any participant deaths, we will also tag their NHS records to ensure we capture any cardiac- and non-cardiac-related deaths between baseline and the 8-month follow-up. At the last follow-up we will also request that the date and last recorded known cardiac risk factors in CCRP records are extracted. At the same time we will contact the participant's GP to request that they extract the same data from primary care records, and that they summarise the incidence of any new cardiac events, i.e. death and/or hospital admissions for ACS or revascularisation procedures (CABG or PCI), or mental health events (e.g. selfharm, suicidality) arising since study enrolment. In addition to being outcomes, these data will be part of the safety monitoring of serious adverse events (SAEs) and adverse events (AEs).

The baseline and follow-up interviews will include self-reported cardiac- and non-cardiac- related morbidity and smoking status, antidepressant medication use [66], and resource use. Depressive symptoms will be measured using the BDI-II $[67,68]$, a 21 -item selfreport instrument measuring symptom severity with an emphasis on affective and cognitive symptoms. Higher scores represent greater depression severity (range 0-63), and minimal (0-13), mild (14-19), moderate (20-28) and severe (29-63) symptom severity ranges have been specified. The Beck Anxiety Inventory [69, 70] will be applied as anxiety is commonly comorbid with depression. This 21-item, self-report measure asks patients to report anxiety symptoms over the last week from 0 (none: it did not bother me at all), 1 (mildly: it did not bother me much), 2 (moderately: it was very unpleasant, but I could stand it), and 3 (severely: I could barely stand it). Item scores are summed with total scores of $8-15,26-25$, and 26-63 taken as cut-off points defining categories for mild, moderate, or severe anxiety respectively. Health-related quality of life will be assessed using both generic and disease-specific measures. The 5-item EuroQoL (EQ5D) [71] is a standardised generic measure of healthrelated quality of life, which is suitable for use in people with a wide range of health conditions, and is recommended by NICE for economic evaluations alongside clinical trials. We will also administer the HeartQoL [72, 73], a tool comprising 14 items, with 10-item physical and 4-item emotional subscales, which are scored from 0 (poor health-related quality of life) to 3 (better). To assess when and how participants become activated over the course of their EPC, we will administer the 9item Behavioral Activation for Depression Scale - Short Form (BADS-SF) [74] at baseline, and the 5-month and 8-month follow-up assessments. The BADS-SF is a 9item tool, where each item is rated from 0 (not at all) to 6 (completely). The total score ranges from 0 to 54, with high scores representing greater activation. Finally, participant experiences of care will be assessed at the 5-month follow-up only, using the 8-item, self-reported Client Satisfaction Questionnaire (CSQ-8) [75] and an adapted version of the NHS Friends and Family Test (FFT). The CSQ items use a 4-point Likert scale (1 to 4) response set, with the total scores ranging from a possible 8 to 32; and higher values indicating greater satisfaction. The FFT consists of a single item with a 5point rating scale ('Extremely likely' to 'Extremely unlikely') and two free-text items ('What was good about your experience?' and 'What would have made your experience better?').

\section{Economic evaluation}

The analytical perspective of NICE's decision-making is a societal costing perspective. The pilot study will test methods of resource and service use data collection enabling estimation of the costs to the NHS, costs to social care and PSS, and relevant costs to patients and their carers/families (including NHS and privately funded mental health care). Wherever feasible, these will distinguish use of services for (1) depression or anxiety; (2) for other mental health problems; (3) for other health reasons or social needs. A preliminary assessment of the cost of providing EPC within CR will be undertaken. Service use data will be collected from routine/administrative sources (e.g. hospital records, GP records, community mental health 
team records, social care records) and participant selfreport using the Service and Resource Use Questionnaire (SRUQ). The SRUQ will be adapted from the Client Services Receipt Inventory [76], with input from our lay advisors. The completeness, validity and reliability of participant-reported versus routine administrative data will be compared in relation to the types/amounts of health care/service used over the follow-up periods planned for a definitive trial.

\section{Statistical analysis plan}

Trial data will estimate CR team recruitment and patient study completion rates. As this is a pilot study we will not report definitive estimates of effectiveness and costs; our primary aim is to document the adequacy of trial procedures, intervention acceptability and outcome measures. All pilot study data will be collected [77] and reported according to the CONSORT extension for cluster randomised trials [78]. Recruitment, intervention and control uptake, outcome completion rates, and drop out will be reported (with $95 \%$ CIs). We will compare the recruitment rate and participant characteristics at baseline between the trial arms to assess whether there is evidence of selection bias resulting from (cluster) randomising teams before participant recruitment [79]. Patient outcome data (including resource use) will be analysed descriptively (e.g. means, standard deviations (SDs)) for each arm at each followup and we will report outcome effect sizes (i.e. betweengroup mean differences and $95 \% \mathrm{CIs}$ ) based on an intention-to-treat analysis. We will explore the impact of patient preferences documented at baseline in the analysis. We will also conduct an exploratory sensitivity analysis reporting between-group outcomes in the sub-groups of patients who do, or do not attend, cardiac rehabilitation programmes.

\section{Qualitative interview study Data collection}

Several methods of data collection will be used. The researcher will observe staff training to identify what issues nurses appear to struggle with and what practical concerns they have about EPC. Observations will be recorded through extensive field notes. Nurses trained in EPC will be invited to take part in an interview towards the end of EPC delivery (once they have gained experience in delivering the intervention), to explore their views on the nurse manual, and to assess their views and experiences of delivering EPC within existing programmes. These interviews will be held by an experienced qualitative interviewer (RW) following a topic guide. Interviews will be conducted either by telephone or on a face-to-face basis, as the teams will be based across a large geographical area and telephone interviews can be used to gather the same material as those held face-toface [80]. Written consent will be secured from those interviewed in person and verbal consent will be gained from those interviewed by telephone. It is predicted that up to 15 nurses will be involved in delivery of EPC in the five CCRPs allocated to the intervention arm. The aim will be to interview around 10 of these nurses.

In-depth interviews will be conducted with patients recruited to the trial who subsequently agree to take part in qualitative interviews. When sampling these patients we will aim for maximum variation in relation to trial arm, age, CR team, gender, socio-economic background, depression score at baseline and adherence to the intervention. Participants will be interviewed shortly after they have completed their 5-month follow-up measurements in case the interview process influences their views of the intervention or trial. Participants will be given an opportunity to ask questions before the interview takes places, and written informed consent will be obtained prior to commencing. All interviews will be conducted by RW following a topic guide. Individuals will be interviewed by telephone or on a face-to-face basis, and verbal or written consent secured respectively. It is predicted that about 30 participants recruited to the trial will be interviewed (10 usual care, 12 completers and 8 non-completers of EPC).

Interviews will also be held with patients who declined to take part in the trial to identify reasons for their decision. These interviews will usually be held by telephone to encourage participation. The data gathered will indicate ways to maximise recruitment and to reflect upon how representative individuals in the trial are of other $\mathrm{CR}$ patients. About 10 'decliners' will be interviewed early on in the trial.

\section{Qualitative analysis plan}

Data collection and analysis will proceed in parallel. All interview data will be audio-recorded, transcribed verbatim and analysed thematically with the aid of NVivo, and using an approach based on a framework approach [81]. Analysing the data thematically will enable comparisons to be made within and across the interviews, and for the views of staff and patients to be compared and contrasted regarding specific topics, such as intervention acceptability.

\section{Ethical and governance considerations}

The Royal Devon and Exeter NHS Foundation Trust is acting as the trial sponsor. This paper describes the CADENCE study protocol (version 4, 19 June 2015), which was reviewed and a favourable ethical opinion obtained from the National Research Ethics Service (NRES) Committee South West - Exeter (reference: 14/ SW/0139) and the relevant NHS research governance 
approvals were obtained prior to commencing fieldwork from: the Royal Cornwall Hospitals NHS Trust; Somerset Partnership NHS Foundation Trust; Taunton and Somerset NHS Foundation Trust; Torbay and South Devon NHS Foundation Trust; University Hospitals Bristol NHS Foundation Trust; University Hospitals Coventry and Warwickshire NHS Trust; Weston Area Health Trust; and Yeovil District Hospital NHS Foundation Trust. Any protocol amendments will be notified through existing pathways in the UK NRES and NHS research governances system, and the trial team will ensure that all participating CCRPs, staff and patients, and trial registries are informed of any changes to the protocol.

This study is registered with a trials registry (ISRCTN34701576) and has been adopted by the UK Comprehensive Research Network (UKCRN ID: 17105). All researchers and nurses involved in participant recruitment will undertake Good Clinical Practice (GCP) training.

\section{Data management}

All personal information obtained about patients or staff for the purposes of recruitment or data collection will be held in accordance with the UK Data Protection Act, 1998. Each participant will be assigned a research number and all outcome data will be encrypted and stored without the participant's name or address. Electronic study records will be stored in a SQL server database managed by the Peninsula Clinical Trial Unit (PenCTU), hosted on a secure server maintained by Plymouth University. Access to electronic data will be permissionbased, with access to identifiable information limited to those processing questionnaires and performing initial screening activities. Copies of study data retained at participating sites will be securely stored for the duration of the study prior to archiving. Electronic copies of any letters or e-mails sent to study participants will be stored securely on a password-protected computer at the University of Exeter, and paper-based information held in a locked filing cabinet in the research team offices. Names and participant details will not be passed onto any third parties and no named individuals will be included in the write-up of the results. The only time personal information would be passed on to a third party (i.e. the patient's GP) would be if we considered there to be risk of serious harm to a research participant and safety protocols need to be activated.

All study data will be kept for 10 years under secure conditions on University of Plymouth and University of Exeter servers. We will seek consent from participants for core outcome data from the pilot study to be released in an anonymised form to an independent data repository (for example, the UK Data Archive) following publication of the key planned outcome papers.

\section{Safety of participants and researchers}

While we believe the study has minimal implications for health care staff, participants will have been found to have depressive symptoms and it is, therefore, appropriate to have a safety policy in place regarding the management of potentially serious adverse events (e.g. selfinjury or suicidality). The Participant Information Sheet will let the person know that if we are very concerned about their safety or someone else's safety we may need to break confidentiality and inform their GP.

Involvement in research interviews where participants are asked to reflect on their health issues can, in some cases, cause distress. Should any such difficulties occur during a research interview, the researcher will offer support to the person involved. Should a participant appear significantly upset and at risk as a result of the interview, the researcher will (with permission) advise the individual's GP of this distress and encourage the person to seek further support from the support network available to them. Should there be any concern that a participant is likely to cause harm either to themselves or another person, their GP will be notified immediately by a senior clinician from the study team. Since researchers will be conducting interviews alone with participants, potentially in the participant's own home, it will be important to have safeguards in place to protect both participant and researcher. Disclosure and Barring Service checks will be performed on any researcher taking part to ensure that they are an appropriate person to be working with vulnerable adults. To ensure the safety of researcher and patient, a lone-worker policy will be applied, providing a mechanism for ensuring that the exact whereabouts of researchers and patients engaged in research activities is known.

\section{Patient and public involvement}

Patient and public involvement (PPI) has been sought during protocol development. The NIHR CLAHRC for the South West Peninsula (PenCLAHRC) hosts the Peninsula PPI Group (http://clahrc-peninsula.nihr.ac.uk/patient-and-public-involvement-in-research). This group has published a framework regarding PPI involvement in research [82], and provides research training and support to facilitate the public becoming actively involved in all aspects of the research process. The co-applicant (AG, PPI facilitator) recruited four lay advisors with relevant, lived experience, each of whom reviewed and commented on our research proposal. PPI will continue throughout this project, with the precise roles of advisors negotiated across time. In the early stage, our lay advisors reviewed outcome measures and informed the design of all study-related materials, e.g. information sheets and consent forms. Our lay advisors were also involved in the development of the intervention 
and accompanying training manual. This helped ensure the acceptability of the intervention to patients. Four lay advisors also attend our project management meetings on a quarterly basis and actively participate in decisions related to the ongoing running of the project. A key area of future involvement will be in dissemination plans. Our lay advisors will work with us to proactively shape the BACPR standards, ensuring that the needs of patients with depression are embedded within the core service descriptions. When sitting on committees, lay advisors will have equal voting rights compared with academic/clinical members. Lay advisors will be paid costs for travel and for time spent at meetings in line with INVOLVE guidance on good practice. AG will support lay advisors attending meetings and/or identify appropriate training opportunities. This will maximise the ability of our lay representatives to actively contribute to our work.

\section{Project oversight}

JC (chief investigator) and SR (scientific lead) will supervise and oversee the strategic development and progress of the project, liaising with the wider team and methodological leads on a routine basis. All research fellows will be based in Exeter (with JC and SR) and will manage the day-to-day aspects of the research relevant to their own role, supported by an administrator at peak periods of fieldwork activity. KT will provide qualitative expertise and supervision, RA is the health economics lead, OCU is the statistics lead, and AG will lead PPI engagement. Monthly project management meetings will be organised to ensure co-applicants and two lay advisors are kept informed of progress and contribute to the ongoing steering of the research.

As this is a pilot trial, a data monitoring committee has not been convened. A Trial Steering Committee (TSC) with independent chair will be appointed in accordance with MRC guidelines. Members will represent an appropriate range of perspectives and expertise (e.g. two lay advisors, clinicians, methodologists), meeting at critical points in the project timeline. The TSC will monitor the scientific quality, ethics and general progress of the project, and will monitor the SAE/AE data emerging from the study.

\section{Project timeline}

The project commenced on 1 April 2014 and is scheduled to complete in July 2016. The feasibility study was reported in May 2015. Preparatory work for the pilot trial, including CR team recruitment, commenced January 2015. Cluster randomisation took place in April 2015, and intervention training delivered to the five $C R$ teams in June-July 2015. Participant recruitment commenced July 2015 and is scheduled to complete by the end of 2015 .
Follow-up data and qualitative interviews will take place between September 2015 and June 2016. Data analysis will commence in January 2016, with the final report due in July 2016.

\section{Discussion}

This paper presents the study protocol (version 4, 19 June 2015) for the CADENCE study, reported to satisfy the Standard Protocol items: Recommendations for Interventional Trials (SPIRIT) guidelines (Additional file 2). This study will provide important information supporting the development and subsequent evaluation of psychological treatment for patients identified with depressive symptoms following an ACS or revascularisation procedure. Our EPC intervention involves training $C R$ nurses in the application of evidence-based mental health care co-ordination algorithms, and in how to actively support and monitor patients working through a BA self-help manual with associated tasks. While both mental health care co-ordination and BA have previously been demonstrated to be effective treatments for patients with depression, their application to busy CR settings and patients is untested. A crucial element of this research will be to examine the feasibility and acceptability of implementing mental health care management strategies within the routine care. Study outcomes will inform the design of a definitive clinical trial that will provide evidence of the effectiveness, cost-effectiveness and acceptability of embedding psychological care within the CR care pathway.

This study was funded in response to a commissioning brief from the Health Technology Assessment board of the UK the National Institute for Health Research (NIHR; project reference 12/189/06), which identified this research area as an important knowledge gap and which sought to investigate an intervention embedded within routine care. We will disseminate our findings widely, to ensure this research will guide policy-makers and clinicians in the future development and organisation of psychological care for patients with depressive symptoms engaging with $\mathrm{CR}$ services. We anticipate that data on acceptability will be of immediate interest to patients and their families, professionals responsible for the design and implementation of $C R$ services, and to the professional body (BACPR) responsible for defining core services and standards of care within NHS CR services.

\section{Trial status}

Patient participant recruitment commenced in July 2015 and is ongoing, scheduled for completion in December 2015. 


\section{Additional files}

\section{Additional file 1: Guide for session planning for nurses delivering enhanced psychological care as part of a comprehensive cardiac rehabilitation programme. (DOCX $44 \mathrm{~kb}$ )}

Additional file 2: SPIRIT Checklist: CADENCE study protocol (version 4, 19 June 2015). (DOCX $61 \mathrm{~kb}$ )

\begin{abstract}
Abbreviations
ACS: acute coronary syndrome; ADM: antidepressant medication; AE: adverse event; BA: behavioural activation; BACPR: British Association for Cardiovascular Prevention and Rehabilitation; BADS-SF: Behavioral Activation for Depression Scale - Short Form; BDI-II: Beck Depression Inventory; BMI: body mass index; CABG: coronary artery bypass grafting; CBT: cognitive behavioural therapy; CIS-R: revised clinical interview schedule; CR: cardiac rehabilitation; CRRP: comprehensive cardiac rehabilitation programme; CSQ-8: Client Satisfaction Questionnaire (CSQ-8); EQ-5D: EuroQoL Index; FFT: Friends and Family Test; GP: general practitioner; IAPT: Improving Access to Psychological Therapies; ICC: intra-cluster correlation coefficient; MI: myocardial infarct; MICD: minimally important clinical difference; NHS: National Health Service; NICE: National Institute of Clinical Excellence; NIHR: National Institute for Health Research; PCl: percutaneous coronary intervention; PHQ-9: Patient Health Questionnaire-9; PPI: patient and public involvement; SAE: serious adverse event: SRUQ: Service and Resource Use Questionnaire.
\end{abstract}

\section{Competing interests}

RST is an author on a number of other Cochrane reviews of cardiac rehabilitation and co-chief investigator on the REACH-HF programme of research, which is developing and evaluating a home-based CR intervention for people with heart failure and their carers (NIHR PGFAR RP-PG-0611-12004). None of the other authors have any competing interests to declare.

\section{Authors' contributions}

JC was chief investigator and co-conceived and designed the study with the involvement of SHR, CD and DAR. MG, LK and RST provided clinical expertise regarding cardiology and cardiac rehabilitation service organisation and $C D$ DK and WK designed the enhanced psychological care intervention under the lead of DAR. SHR was the scientific lead and drafted the study protocol. SHR and CW jointly secured ethics and NHS research and development approvals with input from RW and AD, and the methodological leads for qualitative (KT), statistical (OU), economic (RA) and PPI (AG) elements of the study design. The study researchers (CW, RW) drafted materials to support the study implementation and ethic review, and developed methods to ensure successful implementation of the study protocol. The study researchers (AD, RW, CW) will collect the patient and nurse data. FW is the trial analyst, contributing to the development of both statistical and economic analysis plans. SHR drafted the manuscript. All authors contributed to editing of the final manuscript and the refining of its intellectual content. All authors read and approved the final manuscript.

\section{Acknowledgements}

This project was funded by the UK NIHR Health Technology Assessment Programme (project number 12/189/06). The Royal Devon and Exeter NHS Foundation Trust is acting as trial sponsor. The Funding Agency and Trial sponsors have not been substantively involved in the design, or data acquisition for this research, nor the drafting of this manuscript; the views and opinions expressed in this paper are those of the authors and do not necessarily reflect those of the Health Technology Assessment Programme, NIHR, NHS or the Department of Health.

Rob Anderson, John Campbell, Christopher Dickens, David Richards, Suzanne Richards, Rod Taylor, Obioha Ukoumunne, and Fiona Warren are centrally funded by the University of Exeter Medical School. David Kessler and Katrina Turner are funded by the University of Bristol, Andrew Gibson by the University of the West of England, and Willem Kuyken by Oxford University. Manish Gandhi and Luke Knight are funded by the Royal Devon and Exeter NHS Foundation Trust. Antoinette Davey, Rachel Winder and Christine Wright are employed by the University of Exeter Medical School on the above funding award. David Richards, Rod Taylor and Obioha Ukoumunne are also supported by the NIHR Collaboration for Leadership in Applied Health Research and Care South West Peninsula.
We would like to thank the external members of the CADENCE Trial Steering Committee for their ongoing advice and support for the project: Professor Glyn Lewis (University College London), Professor Anne Rogers (Southampton University), Professor Gill Furze (Coventry University, President of BACPR), Dr Sally Kerry (Queen Mary University of London), Rev Paul Lanham (patient representative), Mr Ron Grant (patient representative). We are also grateful to our Patient and Public Involvement group for their insightful advice and input throughout the trial.

We would like to thank both the staff and patients of our participating CR programmes who are giving generously of their time to support the study and complete data collection materials. We also thank staff from the NIHR Clinical Research Network South West Peninsula and our sponsors, the Royal Devon and Exeter NHS Foundation Trust, who continue to provide us with valuable advice and support in the conduct of this trial.

\section{Author details}

'University of Exeter Medical School, St Luke's Campus, Exeter, EX1 2LU, UK. ${ }^{2} \mathrm{NIHR}$ Collaboration for Leadership in Applied Health Research and Care for the South West Peninsula, University of Exeter Medical School, St Luke's Campus, Exeter EX1 2LU, UK. ${ }^{3}$ School of Social and Community Medicine, University of Bristol, Oakfield House, Oakfield Grove, Clifton, Bristol BS8 2BN, UK. ${ }^{4}$ School of Social and Community Medicine, University of Bristol, Canynge Hall, Whatley Road, Bristol BS8 2PS, UK. ${ }^{5}$ University Department of Psychiatry, University of Oxford, Warneford Hospital, Oxford 0X3 7JX, UK. ${ }^{6}$ Royal Devon and Exeter NHS Foundation Trust, Barrack Road, Exeter EX2 5DW, UK. ${ }^{7}$ Health and Social Sciences, University of the West of England, Frenchay Campus, Coldharbour Lane, Bristol BS16 1QY, UK.

Received: 4 November 2015 Accepted: 16 January 2016

Published online: 02 February 2016

\section{References}

1. Thombs BD, Ziegelstein RC, Pilote L, Dozois DJ, Beck AT, Dobson KS, et al. Somatic symptom overlap in Beck Depression Inventory-II scores following myocardial infarction. Br J Psychiatry. 2010;197(1):61-6. doi:10.1192/bjp.bp.109.076596.

2. Connerney I, Shapiro PA, McLaughlin JS, Bagiella E, Sloan RP. Relation between depression after coronary artery bypass surgery and 12-month outcome: a prospective study. Lancet. 2001;358(9295):1766-71. doi:10.1016/S0140-6736(01)06803-9.

3. Lesperance F, Frasure-Smith N, Juneau M, Theroux P. Depression and 1-year prognosis in unstable angina. Arch Intern Med. 2000;160(9):1354-60.

4. Rutledge T, Reis VA, Linke SE, Greenberg BH, Mills PJ. Depression in heart failure a meta-analytic review of prevalence, intervention effects, and associations with clinical outcomes. J Am Coll Cardiol. 2006;48(8):1527-37. doi:10.1016/j.jacc.2006.06.055

5. Singleton N, Bumpstead R, O'Brien M, Lee A, Meltzer H. Psychiatric morbidity among adults living in private households, 2000. London: HMSO2001 Feb-May. Report No.: 0954-0261 Contract No.: 1-2.

6. Rugulies R. Depression as a predictor for coronary heart disease. a review and meta-analysis. Am J Prev Med. 2002;23(1):51-61.

7. Wulsin LR, Singal BM. Do depressive symptoms increase the risk for the onset of coronary disease? A systematic quantitative review. Psychosom Med. 2003;65(2):201-10.

8. Dickens C, McGowan L, Percival C, Douglas J, Tomenson B, Cotter L, et al. Association between depressive episode before first myocardial infarction and worse cardiac failure following infarction. Psychosomatics. 2005;46(6): 523-8. doi:http://dx.doi.org/10.1176/appi.psy.46.6.523.

9. Leung YW, Flora DB, Gravely S, Irvine J, Carney RM, Grace SL. The impact of premorbid and postmorbid depression onset on mortality and cardiac morbidity among patients with coronary heart disease: meta-analysis. Psychosom Med. 2012;74(8):786-801.

10. Dickens CM, Percival C, McGowan L, Douglas J, Tomenson B, Cotter L, et al. The risk factors for depression in first myocardial infarction patients. Psychol Med. 2004;34(6):1083-92.

11. Dickens C, McGowan L, Percival C, Tomenson B, Cotter L, Heagerty A, et al. Negative illness perceptions are associated with new-onset depression following myocardial infarction. Gen Hosp Psychiatry. 2008;30(5):414-20.

12. Dickens $C$. Depression in people with coronary heart disease: prognostic significance and mechanisms. Curr Cardiol Rep. 2015:17(10):1-10. doi:10.1007/s11886-015-0640-6. 
13. Dickens CM, McGowan L, Percival C, Tomenson B, Cotter L, Heagerty A, et al. Contribution of depression and anxiety to impaired health-related quality of life following first myocardial infarction. Br J Psychiatry. 2006;189:367-72. doi:10.1192/bjp.bp.105.018234.

14. Dickens C, Cherrington A, McGowan L. Depression and health-related quality of life in people with coronary heart disease: a systematic review. Eur J Cardiovasc Nurs. 2012;11(3):265-75. doi:10.1177/ 1474515111430928.

15. Barth J, Schumacher M, Herrmann-Lingen C. Depression as a risk factor for mortality in patients with coronary heart disease: a meta-analysis. Psychosom Med. 2004;66(6):802-13. doi:10.1097/01.psy.0000146332.53619.b2.

16. Nicholson A, Kuper $H$, Hemingway $H$. Depression as an aetiologic and prognostic factor in coronary heart disease: a meta-analysis of 6362 events among 146538 participants in 54 observational studies. Eur Heart J. 2006; 27(23):2763-74

17. van Melle JP, de Jonge P, Spijkerman TA, Tijssen JG, Ormel J, van Veldhuisen DJ, et al. Prognostic association of depression following myocardial infarction with mortality and cardiovascular events: a meta-analysis. Psychosom Med. 2004;66(6):814-22. doi:10.1097/01.psy.0000146294.82810.9c.

18. Meijer A, Conradi HJ, Bos EH, Thombs BD, van Melle JP, de Jonge P. Prognostic association of depression following myocardial infarction with mortality and cardiovascular events: a meta-analysis of 25 years of research. Gen Hosp Psychiatry. 2011;33(3):203-16.

19. Dickens C, Katon W, Blakemore A, Khara A, McGowan L, Tomenson B, et al. Does depression predict the use of urgent and unscheduled care by people with long term conditions? A systematic review with meta-analysis. J Psychosom Res. 2012;73(5):334-42. doi:10.1016/j.jpsychores.2012.08.018.

20. Frasure-Smith N, Lesperance F, Gravel G, Masson A, Juneau M, Talajic M, et al. Depression and health-care costs during the first year following myocardial infarction. J Psychosom Res. 2000;48(4-5):471-8.

21. Rutledge $T$, Vaccarino $V$, Johnson BD, Bittner $V$, Olson MB, Linke SE, et al. Depression and cardiovascular health care costs among women with suspected myocardial ischemia: prospective results from the WISE (Women's Ischemia Syndrome Evaluation) Study. J Am Coll Cardiol. 2009;53(2):176-83.

22. de Jonge $P$, van den Brink RH, Spijkerman TA, Ormel J. Only incident depressive episodes after myocardial infarction are associated with new cardiovascular events. J Am Coll Cardiol. 2006;48(11):2204-8. doi:10.1016/j. jacc.2006.06.077.

23. Dickens C, McGowan L, Percival C, Tomenson B, Cotter L, Heagerty A, et al, New onset depression following myocardial infarction predicts cardiac mortality. Psychosom Med. 2008;70(4):450-5. doi:10.1097/PSY. Ob013e31816a74de.

24. Parker GB, Hilton TM, Walsh WF, Owen CA, Heruc GA, Olley A et al. Timing is everything: the onset of depression and acute coronary syndrome outcome. Biological Psychiatry. 2008;:64(8):pp. doi:10.1016/j.biopsych.2008. 05.02118602090.

25. NICE commissioning guides. Cardiac rehabilitation services. London 201301 November 2013

26. British Association for Cardiovascular Prevention and Rehabilitation. The BACPR standards and core components for cardiovascular disease prevention and rehabilitation 2012. London, UK: British Cardiovascular Society ; 2012

27. NACR. National Audit of Cardiac Rehabilitation: 2012. London, UK: British Heart Foundation; 2012.

28. Dickens C, McGowan L, Percival C, Tomenson B, Cotter L, Heagerty A, et al. Depression is a risk factor for mortality after myocardial infarction: fact or artifact? J Am Coll Cardiol. 2007;49(18):1834-40. doi:10.1016/j.jacc.2007.01.075

29. National Collaborating Centre for Mental Health. Depression in adults with a chronic physical health problem (NICE Clinical Guideline 91). London: National Institute for Health and Clinical Excellence; 2009

30. National Collaborating Centre for Mental Health. Depression: the treatment and management of depression in adults (NICE Clinical Guideline 90). London: National Institute for Health and Clinical Excellence; 2009. Report No.: 9781849360999 (pbk.).

31. National Collaborating Centre for Mental Health. Common mental disorders: the NICE guidance on identification and pathways to care (NICE Guideline 123). London: National Institute for Health and Clinical Excellence; 2009. Report No:: 9781849360999 (pbk.)

32. Bird A. We need to talk: the case for psychological therapy on the NHS. UK: Mental Health Foundation; 2006.
33. DeRubeis RJ, Hollon SD, Amsterdam JD, Shelton RC, Young PR, Salomon $\mathrm{RM}$, et al. Cognitive therapy vs medications in the treatment of moderate to severe depression. Arch Gen Psychiatry. 2005;62(4):409-16.

34. Barnes M, Sherlock S, Thomas L, Kessler D, Kuyken W, Owen-Smith A, et al. No pain, no gain: depressed clients' experiences of cognitive behavioural therapy. Br J Clin Psychol. 2013;52(4):347-64. doi:10.1111/bjc.12021.

35. Layard R. The case for psychological treatment centres. Br Med J. 2006; 332(7548):1030-2.

36. London School of Economics. The Depression Report. A new deal for depression and anxiety disorders. London: London School for Economics; 2006.

37. Jacobson NS, Martell CR, Dimidjian S. Behavioral activation treatment for depression: returning to contextual roots. Clinical Psychology: Science and Practice. 2001;8(3):255-70. doi:10.1093/clipsy.8.3.255.

38. Addis M, Martell C. Overcoming depression one step at a time: the new behavioral activation approach to getting your life back. Oakland, CA, US : New Harbinger Publications ; 2004.

39. Beck A, Rush AJ, Shaw B, Emery G. Cognitive therapy of depression: a treatment manual. The Guilford clinical psychology and psychotherapy series. New York: Guilford Press; 1979.

40. Ekers D, Richards D, McMillan D, Bland JM, Gilbody S. Behavioural activation delivered by the non-specialist: phase II randomised controlled trial. $\mathrm{Br}$ J Psychiatry. 2011;198(1):66-72. doi:10.1192/bjp.bp.110.079111.

41. Richards D. Behavioural activation. In: Bennett-Levy J, Richards D, Farrand P, Christensen H, Griffiths K, Kavanagh D, et al., editors. Oxford guide to low intensity CBT interventions. Oxford: Oxford University Press; 2010. p. 141-50.

42. Richards D, Farrand P, Chellingsworth M. National Curriculum for the Education of Psychological Wellbeing Practitioners (PWPS). Lancashire: British Association for Behavioural \& Cognitive Psychotherapies; 2011.

43. Richards $D$, Whyte $M$. Reach Out: National programme educator materials to support the delivery of training for practitioners delivering low intensity interventions. London: Rethink, National Mental Health Development Unit, 2009.

44. Lespérance F, Frasure-Smith $N$, Koszycki D, Laliberté M, et al. Effects of citalopram and interpersonal psychotherapy on depression in patients with coronary artery disease: the Canadian cardiac randomized evaluation of antidepressant and psychotherapy efficacy (create) trial. JAMA. 2007;297(4): 367-79. doi:10.1001/jama.297.4.367.

45. Glassman AH, O'Connor CM, Califf RM, Swedberg K, Schwartz P, Bigger Jr JT, et al. Sertraline treatment of major depression in patients with acute MI or unstable angina. JAMA. 2002;288(6):701-9.

46. van Melle JP, de Jonge $\mathrm{P}$, Honig A, Schene AH, Kuyper AM, Crijns HJ, et al. Effects of antidepressant treatment following myocardial infarction. $\mathrm{Br}$ J Psychiatry. 2007;190:460-6.

47. Whalley B, Rees K, Davies P, Bennett P, Ebrahim S, Liu Z, et al. Psychological interventions for coronary heart disease. Cochrane Database Syst Rev. 2011; 8, CD002902. doi:10.1002/14651858.CD002902.pub3.

48. Baumeister H, Hutter N, Bengel J. Psychological and pharmacological interventions for depression in patients with coronary artery disease. Cochrane Database Syst Rev. 2011;9, CD008012. doi:10.1002/14651858. CD008012.pub3.

49. Berkman LF, Blumenthal J, Burg M, Carney RM, Catellier D, Cowan MJ, et al. Effects of treating depression and low perceived social support on clinical events after myocardial infarction: the Enhancing Recovery in Coronary Heart Disease Patients (ENRICHD) Randomized Trial. JAMA. 2003;289(23):3106-16.

50. Whang W, Burg MM, Carney RM, Freedland KE, Bigger JT, Catellier D et al. Design and baseline data from the vanguard of the Comparison of Depression Interventions after Acute Coronary Syndrome (CODIACS) randomized controlled trial. Contemporary Clinical Trials. 2012;33(5):1003-10. doi:http://dx.doi.org/10.1016/j.cct.2012.05.005.

51. Davidson KW, Bigger JT, Burg MM, Carney RM, Chaplin WF, Czajkowski S et al. Centralized, stepped, patient preference-based treatment for patients with post-acute coronary syndrome depression: CODIACS Vanguard Randomized Controlled Trial. Journal of the American Medical Association: Internal Medicine. 2013:1-8. doi:10.1001/jamainternmed.2013.915.

52. Simmonds $R$, Tylee $A$, Walters $P$, Rose D. Patients' perceptions of depression and coronary heart disease: a qualitative UPBEAT-UK study. BMC Fam Pract. 2013;14(1):38

53. Cuijpers $P$, van Straten A, Warmerdam L. Behavioral activation treatments of depression: a meta-analysis. Clin Psychol Rev. 2007;27(3):318-26. doi:10.1016/j.cpr.2006.11.001. 
54. Ekers D, Richards D, Gilbody S. A meta-analysis of randomized trials of behavioural treatment of depression. Psychol Med. 2008;38(5):611-23. doi:10.1017/S0033291707001614.

55. Craig P, Dieppe P, Macintyre S, Michie S, Nazareth I, Petticrew M. Developing and evaluating complex interventions: new guidance: London, UK: Medical Research Council. 2008.

56. Thabane L, Ma J, Chu R, Cheng J, Ismaila A, Rios L, et al. A tutorial on pilot studies: the what, why and how. BMC Med Res Methodol. 2010;10:1.

57. Lancaster GA, Dodd S, Williamson PR. Design and analysis of pilot studies: recommendations for good practice. J Eval Clin Pract. 2004;10(2):307-12. doi:10.1111/j.2002.384.doc.x.

58. Kroenke K, Spitzer RL, Williams JB. The PHQ-9: validity of a brief depression severity measure. J Gen Intern Med. 2001;16(9):606-13.

59. Meader N, Mitchell AJ, Chew-Graham C, Goldberg D, Rizzo M, Bird V, et al Case identification of depression in patients with chronic physical health problems: a diagnostic accuracy meta-analysis of 113 studies. Br J Gen Pract. 2011;61(593):e808-20. doi:10.3399/bjgp11X613151.

60. Lowe B, Unutzer J, Callahan CM, Perkins AJ, Kroenke K. Monitoring depression treatment outcomes with the Patient Health Questionnaire-9. Med Care. 2004;42(12):1194-201.

61. NACR. The National Audit of Cardiac Rehabilitation: annual statistical report 2014. London: British Heart Foundation; 2014.

62. McMillan D, Gilbody S, Richards D. Defining successful treatment outcome in depression using the PHQ-9: a comparison of methods. J Affect Disord. 2010;127(1-3):122-9. doi:10.1016/j.jad.2010.04.030.

63. Lewis $\mathrm{G}$. Assessing psychiatric disorder with a human interviewer or a computer. J Epidemiol Community Health. 1994;48(2):207-10.

64. Lewis G, Pelosi AJ, Araya R, Dunn G. Measuring psychiatric disorder in the community: a standardized assessment for use by lay interviewers. Psychol Med. 1992;22(2):465-86.

65. King M, Nazareth I, Lampe F, Bower $\mathrm{P}$, Chandler M, Morou M, et al. Conceptual framework and systematic review of the effects of participants and professionals' preferences in randomised controlled trials. Health Technol Assess. 2005;9(35):1-186. iii-iv.

66. Morisky DE, Green LW, Levine DM. Concurrent and predictive validity of a self-reported measure of medication adherence. Med Care. 1986;24(1): 67-74.

67. Beck AT, Steer RA, Brown GK. Beck Depression Inventory - second edition: manual. 2nd ed. San Antonio: The Psychological Corporation; 1996.

68. Beck CA, Joseph L, Belisle P, Pilote L. QOLAMI Investigators. Predictors of quality of life 6 months and 1 year after acute myocardial infarction. Am Heart J. 2001;142(2):271-9. doi:10.1067/mhj.2001.116758.

69. Beck AT, Steer RA. Manual for the Beck Anxiety Inventory. San Antonio: The Psychological Corporation; 1990.

70. Beck AT, Steer RA, Ball R, Ciervo CA, Kabat M. Use of the Beck Anxiety and Depression Inventories for primary care with medical outpatients. Assessment. 1997;4(3):211-9.

71. The EuroQol Group. EQ-5D user guide. Rotterdam: The EuroQol Group; 1996.

72. Oldridge N, Hofer S, McGee H, Conroy R, Doyle F, Saner H. The HeartQoL: Part II. Validation of a new core health-related quality of life questionnaire for patients with ischemic heart disease. Eur J Prev Cardiol. 2012. doi:10.1177/2047487312450545

73. Oldridge N, Hofer S, McGee H, Conroy R, Doyle F, Saner H. The HeartQoL: Part I. Development of a new core health-related quality of life questionnaire for patients with ischemic heart disease. Eur J Prev Cardiol. 2012. doi:10.1177/2047487312450544.

74. Manos RC, Kanter JW, Luo W. The behavioral activation for depression scaleshort form: development and validation. Behav Ther. 2011;42(4):726-39. doi:10.1016/j.beth.2011.04.004.

75. Attkisson C, Zwick R. The client satisfaction questionnaire. Psychometric properties and correlations with service utilization and psychotherapy outcome. Eval Program Plann. 1982;5(3):233-7.

76. Beecham J, Knapp M. Costing psychiatric interventions. Measuring mental health needs. London: Gaskell; 1992. p. p. viii, 328 p.

77. Weijer C, Grimshaw J, Eccles M, McRae A, White A, Brehaut J, et al. The Ottawa Statement on the ethical design and conduct of cluster randomized trials. PLoS Med. 2012;9(11), e1001346. doi:10.1371/journal. pmed.1001346.

78. Campbell MK, Piaggio G, Elbourne DR, Altman DG, Group C. Consort 2010 statement: extension to cluster randomised trials. Br Med J. 2012 345 , e5661.
79. Eldridge S, Kerry S, Torgerson DJ. Bias in identifying and recruiting participants in cluster randomised trials: what can be done? Br Med J. 2009; 339:b4006. doi:10.1136/bmj.b4006.

80. Sturges JE, Hanrahan KJ. Comparing telephone and face-to-face qualitative interviewing: a research note. Qual Res. 2004;4(1):107-18. doi:10.1177/1468794104041110.

81. Ritchie J, Spencer L. Qualitative data analysis for applied policy research. In: Bryman A, Burgess RG, editors. Analyzing qualitative data. London: Routledge; 1994. p. 173-94.

82. Gibson A, Britten N, Lynch J. Theoretical directions for an emancipatory concept of patient and public involvement. Health. 2012;16(5):531-47. doi:10.1177/1363459312438563.

83. Department of Health, Strategic Commissioning Development Unit. Cardiac Rehabilitation Commissioning Pack. London: UK Department of Health 2010. http://www.dh.gov.uk/en/Publicationsandstatistics/Publications/ PublicationsPolicyAndGuidance/Browsable/DH_117506.

\section{Submit your next manuscript to BioMed Central and we will help you at every step:}

- We accept pre-submission inquiries

- Our selector tool helps you to find the most relevant journal

- We provide round the clock customer support

- Convenient online submission

- Thorough peer review

- Inclusion in PubMed and all major indexing services

- Maximum visibility for your research

Submit your manuscript at www.biomedcentral.com/submit
) Biomed Central 